\title{
THE SOLAR CORONA ABOVE ACTIVE REGIONS: A COMPARISON OF EXTREME ULTRAVIOLET LINE EMISSION WITH RADIO EMISSION
}

\author{
WERNER M. NEUPERT \\ (Goddard Space Flight Center, Greenbelt, Md., U.S.A.)
}

\begin{abstract}
A BSTRACT
The observations of extreme ultraviolet (EUV) emission lines of FeIx through Fexvi made by OSO-I have been applied to a study of the solar corona above active regions. Ultraviolet and radio emission are determined for several levels of activity classified according to the type of sunspot group associated with the active region. Both radio emission and line radiation from FexvI, the highest stage of ionization of $\mathrm{Fe}$ observed, are observed to increase rapidly with the onset of activity and are most intense over an E spot group early in the lifetime of the active region. As activity diminishes, radiation from Fexv and FexIV becomes relatively more prominent. Preliminary X-ray data from OSO-III obtained during a flare are introduced. These indicate that radiation from the highest stage of iron thus far observed, Fexxv, reaches a maximum first in an X-ray burst and that maxima in lower stages of ionization follow, with delays from 2 to $15 \mathrm{~min}$.
\end{abstract}

\section{Introduction}

With the advent of rockets and satellites, it has become possible to make observations of permitted emission lines originating in the solar corona above active regions as these regions pass across the visible solar disk. The extreme ultraviolet (EUV) observations made by the first Orbiting Solar Observatory (OSO-I), launched on March 7, 1962, presented the first opportunity for such a study over an extended period of time. Contained in the scientific payload of this satellite was a spectrometer (Behring et al., 1962) for recording the solar spectrum in the wavelength range from $\lambda 150$ to $\lambda 400$. The coronal emission lines of many elements were recorded for a time interval greater than 1 year and during a wide range of solar activity (Behring et al., 1963). The observed 27 day variations in line intensities attributable to this activity have been discussed previously (Neupert, 1965).

EUV spectra of the entire Sun have recently been supplemented by slitless spectra (Tousey, 1965), which demonstrate directly the tendency for emission lines from the most highly ionized ions to be most intense over active regions. Because such studies have been made from rockets, no sequential study over the lifetime of any one region has been possible. The present paper attempts to describe the EUV and radio emissions of several specific active regions as they develope with time. 


\section{Active Regions observed by OSO-I}

Our ability to discuss the development of active regions using the OSO-I data is limited by the fact that these observations provided no spatial resolution. The OSO-I EUV spectrometer recorded radiation from the entire visible solar hemisphere at all times, so that the observed counting rate in any spectral line represents the combined emission from all active centers as well as any radiation emitted by the undisturbed solar atmosphere. We have been able to discriminate bet:veen these two components by comparing data taken when the Sun was devoid of any active regions to data taken when one or more active regions were present on the visible solar disk. Minimum EUV line intensities coincided with a Zürich Relative Sunspot number near zero and a minimum in the CaII plage area which occurred on March 9-11. All other EUV data are normalized to the observations of March 9-11. We refer to any increases above a value of one as 'relative enhancements' and assume that they are due to the presence of active regions on the solar disk.

A comparison of these EUV data with concurrent radio observations suggests that the variations of the highest stages of ionization of $\mathrm{Fe}$ which we have observed FeXvI and FeXV - resemble most strongly the radio observations at frequencies around $2000 \mathrm{MHz}$. Emission lines from lower stages of ionization co-relate less well with radio fluxes and show no distinct association with either the higher or the lower range of frequencies although it appears that some fluctuations occurring over a period of only a few days can be traced through successively lower stages of ionization and also through the entire range of radio frequencies.

From these data it is possible to extract two sequences of observations showing changes from month to month in the EUV and radio emission from the corona above active centers. In each of these sequences the active regions were sufficiently similar so that the increases in EUV and radio fluxes could be associated with specific regions of a group of similar regions. The method used will be published in detail in Solar Physics. Results are given in Figure 1. The EUV increase attributed to active regions are again given in units of the flux from the Sun as observed on March 9-11. It should be reiterated that we have used several similar solar regions in arriving at these results. The time scale used in Figure 1 therefore does not apply to any one particular region observed throughout its entire lifetime, although it is correct for the development of one region which was observed in the Southern hemisphere.

The time dependence of the radio emission, as shown in Figure 1, generally confirms earlier observations made by Vauquois (1959). The highest reported frequency $(9400 \mathrm{MHz})$ is emitted almost exclusively during the period of highest activity, while the lowest frequency persists even after sunspots have disappeared. Only weak, or, in most cases, no polarization of the radio emission was observed during the interval of March-May 1962.

Of all the EUV radiations observed by OSO-I, it appears that the fluxes from FexVI 

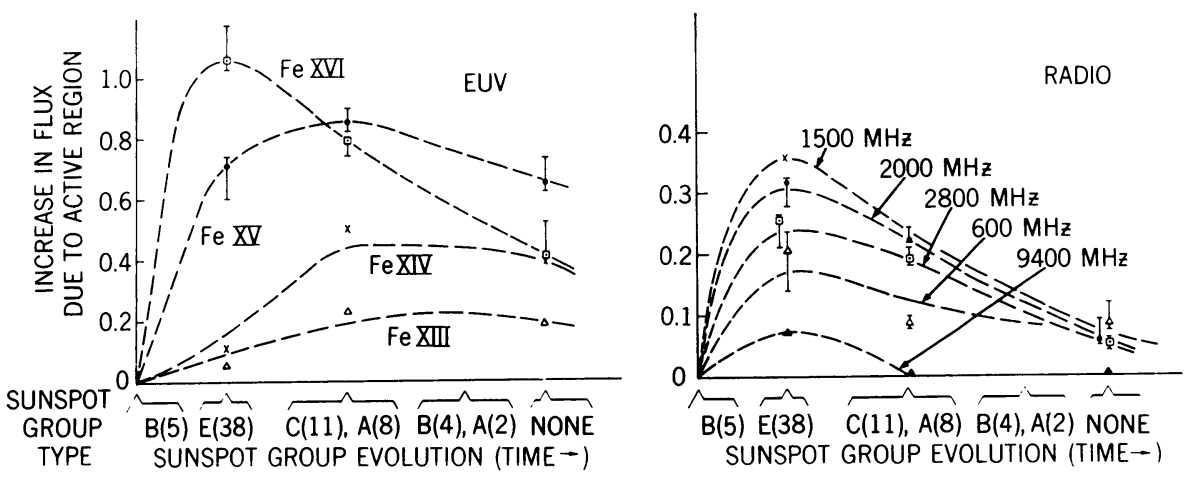

FIG. 1. The EUV and radio fluxes attributed to an active solar region at various stages in its development, as derived from OSO-I observations. The increases in emission are expressed in terms of the total solar emission observed on March 9-11, 1962, in the absence of appreciable solar activity. Note that maximum emission in the highest observed stage of ionization coincides with maximum complexity and size of the sunspot group. The time scale shown is typical for the type of region being discussed. Other such regions may have different rates of development.

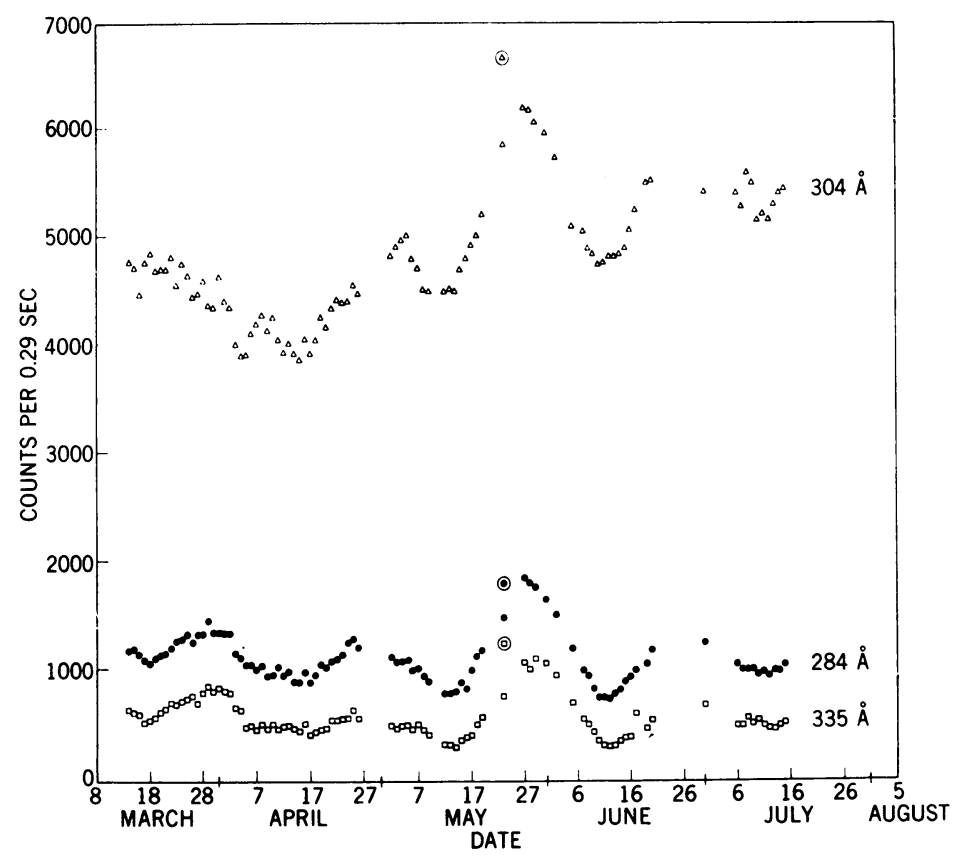

FIG. 2. Preliminary OSO-III data on the counting rates observed for emission lines of Fe XV, Fe XVI and HeII for nearly 5 months of observation starting in March 1967. During this interval, the Zürich Provisional Sunspot Number reached a minimum of 17 on May 10 and on June 10 and 11. A maximum of 197 was attained on May 28. The circled data were obtained during a flare of importance 3 on May 23, 1967. 
and FeXv show the greatest increases over active regions. More precisely, it appears that the greatest increase is associated with the highest stage of ionization, at the time of maximum development of the sunspot group. The similarity of the Fexvi and decimetric radio results extends also to solar flares accompanied by ionospheric effects (Neupert, 1965). In that case the time of maximum emission and total duration of Fexvi emission coincides with the 'gradual rise and fall' observed at decimetric wavelengths. The emission lines from FexIV and FexiII associated with an active region are observed to reach a maximum after the peak of flare activity. Below FexIII, the ultraviolet increases are small and less well-correlated with the appearance of active centers.

The time dependence observed for Fe xIV agrees well with data presented by Le Roy and Trellis at this conference. We ourselves have studied the coronal limb observations at $\lambda 5303$ for March-May 1962, and observe that the forbidden line intensity confirms the dependence on stage of activity as deduced from the EUV data. The results obtained from this work indicate a two-fold advantage in using the EUV observation: (1) Higher stages of ionization, which are more sensitive to solar activity can be

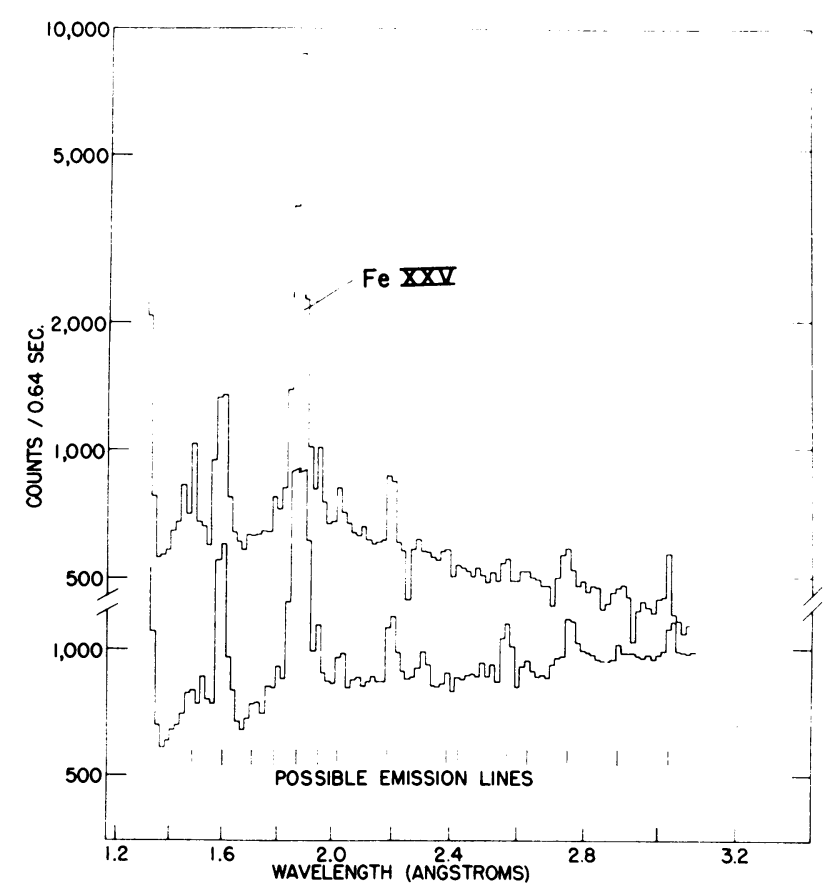

FIG. 3. Two spectral scans in the region $1.3 A$ to $3.1 A$ obtained during the increasing phase of a solar X-ray burst on March 22, 1967. Apparent differences in spectral distribution are due to the increase in intensity of the $X$-ray burst in the time $(5 \mathrm{~min})$ required to make the two scans. The onset of increased count rate at 1.34 A coincides with the detector position at which it begins to be illuminated directly by the Sun. 
observed in the EUV than in the visible region, and (2) The EUV emission can be observed for regions on the disk of the Sun so that the relationship between coronal emission and sunspot activity can be clearly observed for the first time.

\section{Preliminary XUV Observations made by OSO-III}

The Orbiting Solar Observatory III, launched in March, 1967 carries spectrometers, supplied by the Goddard Space Flight Center, for observing the solar spectrum from $1 \AA$ to $400 \AA$. Data for three EUV emission lines obtained with a grating spectrometer are shown in Figure 2. Results concerning active regions appear to be confirmed by these new data. New spectra obtained by OSO-III at shorter wavelengths are shown in Figures 3 and 4. These data were taken during a flare of importance 3 on March 22, 1967. Tentative identifications of strong emission lines of iron have been indicated. A detailed discussion of these identifications has been published elsewhere (Neupert et al., 1967). Of interest in the current discussion is the variation with time of these prominent emission lines. In general we find that the highest stages of ionization rise to maximum the most rapidly and show the greatest amount of variability. Preliminary

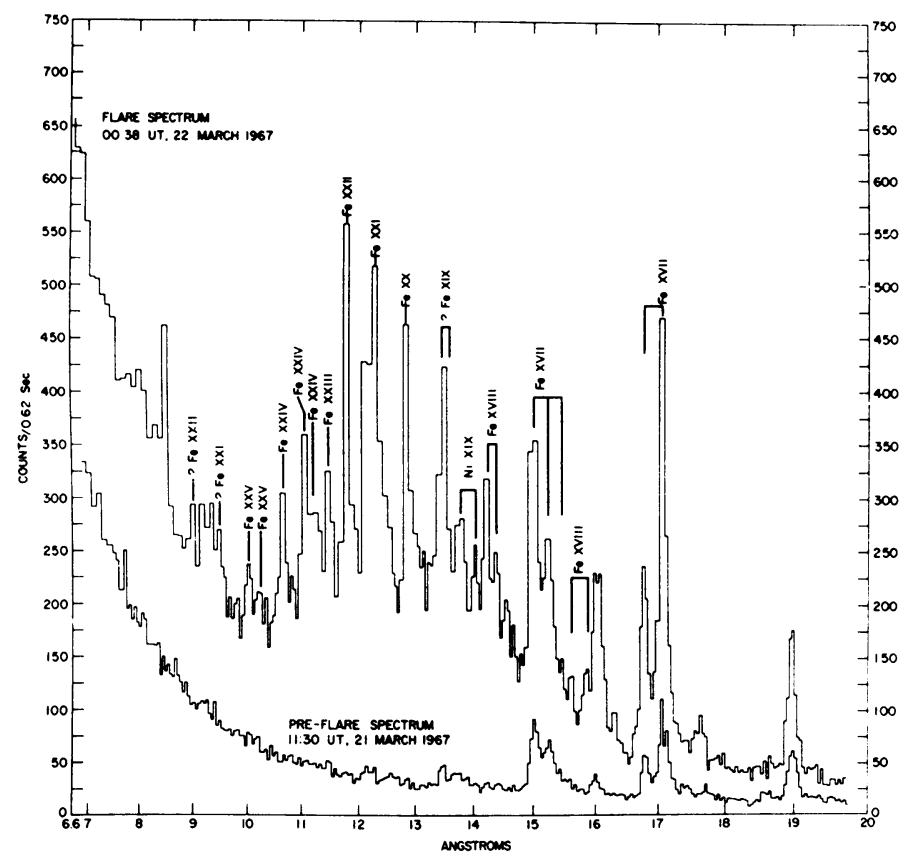

Fig. 4. Comparison of the solar spectrum between $6.3 A$ and $20.0 A$ obtained during a flare on March 22, 1957, with a spectrum obtained on the previous day when no flares were in progress. Tentative identification for new transition arrays of Fe xxv-Fexx are indicated. Spectral resolution is insufficient to allow resolution of lines within each array. Emission lines of $\mathrm{Ni}$ XIX observed in laboratory spectra by Feldman et al. (1967) are also indicated. 
results for the flare of March 22 are given in Figure 5, which shows the strong line at $1.87 \AA$ (Fexxv) reaching maximum intensity earlier than the lines of lower stages of ionization. Thus, at least for some flares, we observe the same characteristic as noted for active regions; that is, that the highest stages of ionization observable tend to increase most rapidly, with lower stages appearing later (compare Figure 1 with Figure 5). Higher stages of ionization are emitted during X-ray bursts than at other times, however.

More detailed studies of intensity with time for these lines, and comparison with the emission in the line of HeII at $304 \AA$, are currently underway.

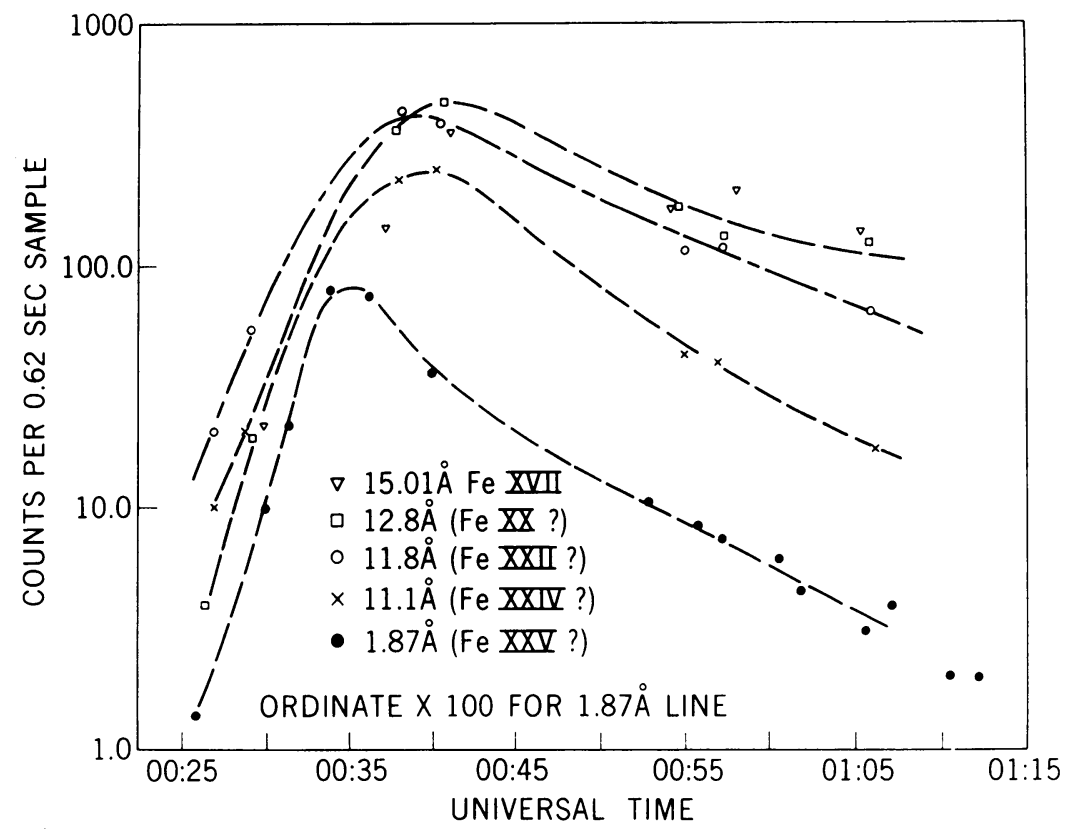

FIG. 5. Preliminary OSO-III observations on the variation of X-ray line emission with time for a flare of importance 3 on March 22, 1967. Note that peak intensity is reached earlier for the higher stages of ionization than for lower stages.

\section{References}

Behring, W.E., Neupert, W. M., Nichols, W. A. (1962) J. Opt. Soc. Am., S2 (5), 597.

Behring, W.E., Neupert, W. M., Lindsay, J.C. (1963) in Space Research, III, Ed. by W. Priester, North-Holland Publishing Co., Amsterdam, p. 814.

Feldman, U., Cohen, L., Swartz, M. (1967) Astrophys. J., 148, 585.

Neupert, W. M. (1965) Ann. Astrophys., 28, 446.

Neupert, W.M., Gates, W., Swartz, M., Young, R. (1967) Ap. J. Letters, 149, No. 2, Part 2, L79. Tousey, R. (1965) Ann. Astrophys., 28, 755.

Vauquois, B. (1959) Paris Symposium on Radio Astronomy, ed. by R. N. Bracewell, Stanford University Press, Stanford, Calif., p. 143. 


\section{DISCUSSION}

Noyes: In the slide showing the variations of $304,335,284 \AA$ lines from OSO-III, is the apparent lesser increase of the 284 line during the flare, compared with the increases of the other two, statistically significant?

Neupert: Each point plotted represents an average of observations made over a period of 1 hour, so that these data do not give the peak emission reached in each line during the flare. The smaller increase in Fexv compared to FexvI was also found for several flares observed in 1962 by the OSO-I satellite.

Krat: In what way have you done your identification of new coronal lines?

Neupert: The first suggestion that iron emission lines might be present was made by Kawabata (Rept. Ion. Space Research Japan, 14, 1960, 405). We have calculated the positions of possible lines (centres of gravity of multiplets) using screening corrections to hydrogenic energy parameters given by Froese (Canadian J. Phys., 41, 1963, 50).

Severny: Have you recorded simultaneously $\mathrm{L} \alpha$-intensity?

Neupert: We have not, but Dr. Hinteregger recorded the region in question.

Newkirk: Would you care to mention electron densities and temperatures which would be consistent with your observations?

Neupert: The bulk of corona material above an active region reaches temperatures up to $4.0 \times 10^{6}{ }^{\circ} \mathrm{K}$ during the period of most intense activity of the region. On the other hand, when the region has subsided the temperature appears to be about $2.5-3.0 \times 10^{6}{ }^{\circ} \mathrm{K}$. Assuming that the area of the region is the same as that of the underlying plage, the electron densities in the active region appear to be about 10 times the quiet corona. These densities decrease to 2-3 times quiet corona values over residual plage after sunspots have disappeared. 